TITLE:

\title{
Tandem trimer pyrrole-imidazole polyamide probes targeting 18 base pairs in human telomere sequences
}

\section{$\operatorname{AUTHOR}(\mathrm{S}):$}

Kawamoto, Yusuke; Sasaki, Asuka; Hashiya, Kaori; Ide, Satoru; Bando, Toshikazu; Maeshima, Kazuhiro; Sugiyama, Hiroshi

\section{CITATION:}

Kawamoto, Yusuke... [et al]. Tandem trimer pyrrole-imidazole polyamide probes targeting 18 base pairs in human telomere sequences. Chemical Science 2015, 6(4): 2307-2312

\section{ISSUE DATE:}

2015-01-20

URL:

http://hdl.handle.net/2433/198452

\section{RIGHT:}

This article is licensed under a Creative Commons Attribution 3.0 Unported Licence. 


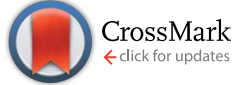

Cite this: Chem. Sci., 2015, 6, 2307

Received 4th December 2014

Accepted 20th January 2015

DOI: $10.1039 / c 4 s c 03755 c$

www.rsc.org/chemicalscience

\section{Tandem trimer pyrrole-imidazole polyamide probes targeting 18 base pairs in human telomere sequences $\dagger$}

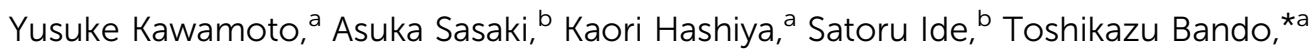 \\ Kazuhiro Maeshima*b and Hiroshi Sugiyama*ac
}

\begin{abstract}
The binding of molecules to specific DNA sequences is important for imaging genome DNA and for studying gene expression. Increasing the number of base pairs targeted by these molecules would provide greater specificity. $\mathrm{N}$-Methylpyrrole- $\mathrm{N}$-methylimidazole $(\mathrm{Py}-\mathrm{Im})$ polyamides are one type of such molecules and can bind to the minor groove of DNA in a sequence-specific manner without causing denaturation of DNA. Our recent work has demonstrated that tandem hairpin Py-Im polyamides conjugated with a fluorescent dye can be synthesized easily and can serve as new probes for studying human telomeres under mild conditions. Herein, to improve their selectivities to telomeres by targeting longer sequences, we designed and synthesized a fluorescent tandem trimer Py-Im polyamide probe, comprising three hairpins and two connecting regions (hinges). The new motif bound to $18 \mathrm{bp}$ dsDNA in human telomeric repeats (TTAGGG) $n$, the longest sequence for specific binding reported for Py-Im polyamides. We compared the binding affinities and the abilities to discriminate mismatch, the UV-visible absorption and fluorescence spectra, and telomere staining in human cells between the tandem trimer and a previously developed tandem hairpin. We found that the tandem trimer Py-Im polyamide probe has higher ability to recognize telomeric repeats and stains telomeres in chemically fixed cells with lower background signal.
\end{abstract}

\section{Introduction}

Telomeres are localized at the ends of chromosomes and play important roles in the stability and replication of chromosomes. ${ }^{1}$ Human telomere DNA, whose sequences are the tandem repeats of $5^{\prime}$-TTAGGG-3' ${ }^{\prime}$, has a duplex region and a $3^{\prime}$ overhang, which tends to form G-quadruplexes or t-loops. ${ }^{2}$ In normal mammalian cells, the number of telomere repeats decreases with cell division, and this decrease is related to the aging process and cancer. ${ }^{3,4}$ Therefore, the telomere length is one important biomarker used in various diagnoses. ${ }^{2-5}$

\footnotetext{
${ }^{a}$ Department of Chemistry, Graduate School of Science, Kyoto University, Kyoto 606-8502, Sakyo, Japan.E-mail: hs@kuchem.kyoto-u.ac.jp; bando@kuchem.kyoto-u. ac.jp

${ }^{b}$ Biological Macromolecules Laboratory, Structural Biology Center, National Institute of Genetics, and Department of Genetics, School of Life Science, Graduate University for Advanced Studies (Sokendai), Mishima, Shizuoka 411-8540, Japan. E-mail: kmaeshim@nig.ac.jp

'Institute for Integrated Cell-Material Science (WPI-iCeMS), Kyoto University, Kyoto 606-8501, Sakyo, Japan

$\dagger$ Electronic supplementary information (ESI) available: Materials and methods, sequences of ODN-1 to 5, mass spectra and analytical HPLC profiles of the compounds TH59, TT59, TAMRA TH59 and TAMRA TT59, the UV-visible absorption and fluorescence spectra of probes TAMRA TH59 and TAMRA TT59, SPR sensorgrams of TH59 and TT59, additional images of telomeres. See DOI: $10.1039 / \mathrm{c} 4 \mathrm{sc} 03755 \mathrm{c}$
}

Fluorescent probes targeting telomeres have been developed to visualize and measure the telomere length, which is regulated by the shelterin complex. ${ }^{6}$

Our group has focused on $N$-methylpyrrole (Py) $-N$-methylimidazole (Im) (Py-Im) polyamides, as reported by Dervan and co-workers. Py-Im polyamides bind strongly and sequencespecifically to the minor groove of dsDNA by recognizing Watson-Crick base pairs without causing DNA denaturation. ${ }^{7}$ The antiparallel Im-Py pair recognizes a $\mathrm{G} \cdot \mathrm{C}$ base pair, whereas a $\mathrm{Py} / \mathrm{Py}$ pair recognizes an $\mathrm{A} \cdot \mathrm{T}$ or $\mathrm{T} \cdot \mathrm{A}$ base pair. ${ }^{7 a, b} \mathrm{C}$-terminal $\beta$-alanine and the $\gamma$-turn moiety comprising $\gamma$-aminobutyric acid or 2,4-diaminobutyric acid (Dab) also recognize A-T or T·A base pairs, and $(R)$-Dab affords a higher binding affinity comparable to that of $\gamma$-aminobutyric acid. ${ }^{8}$

Py-Im polyamides can be synthesized by machine-assisted Fmoc solid-phase peptide synthesis (SPPS). ${ }^{9}$ Various structures of Py-Im polyamides, such as linear, ${ }^{10}$ hairpin, ${ }^{11}$ and cyclic, ${ }^{12}$ have been synthesized to provide efficient binding. In particular, hairpin Py-Im polyamides are versatile and can be used as sequence-specific functional conjugates. ${ }^{13}$ To gain specificity, Py-Im polyamides targeting longer sequences ( $\geq 10 \mathrm{bp}$ ) have been developed..$^{10,14-17}$ DNA ligands recognizing $\geq 16$ bp are required to target a single site in the human genome, which comprises about 3 billion base pairs. Dervan and co-workers reported a linear Py-Im polyamide that formed a homodimer 
with two molecules and then bound to $16 \mathrm{bp}$ sequences in the regulatory region of the HIV-1 genome. ${ }^{10 b}$ Our laboratory has reported a cysteine-derived Py-Im polyamide dimer that binds to $16 \mathrm{bp}$ with one molecule. ${ }^{14 c}$

Tandem hairpin Py-Im polyamides targeting $\geq 10 \mathrm{bp}$, which comprise two hairpin moieties and a hinge segment between two hairpins, have been developed. ${ }^{15,16}$ For instance, Maeshima, Janssen, and Laemmli described tandem hairpin Py-Im polyamide probes that could bind to $12 \mathrm{bp}$ sequences in the duplex region of the human telomere repetitive sequence TTAGGG and could stain telomeres in chemically fixed cells. ${ }^{16}$ Solid-phase synthesis requires many steps, which leads to a lower yield, and there are fewer reports on the targeting longer sequences with Py-Im polyamides than those described for the binding of conventional polyamides to shorter sequences.

Recently, our group proposed a new method for the synthesis of tandem hairpin Py-Im polyamide TH59 (Fig. 1) using a new building block. The block comprises the sequence from Dab to the N-terminus of a hairpin moiety, and is used as a unit of SPPS to decrease the procedure on solid support. Using this methodology, we synthesized fluorescent telomere probes ${ }^{17 a, b}$ and alkylation agents. ${ }^{17 c, d}$ Human telomeres bound by the probes could be costained with anti-TRF1 antibody under mild conditions without breaking the structure of the telomeres, showing that the telomere length was related to the abundance of the shelterin complex. ${ }^{17 a}$ This point is an advantage over the PNA FISH method, which requires denaturation under harsh conditions. ${ }^{6 b}$ More recently, the fluorescent group and the hinge region were optimized to target telomeres in cells more selectively. ${ }^{17 b}$
Herein, as one of the optimizations of the Py-Im polyamide probes, we describe our synthesis of the new type of Py-Im polyamides, defined as tandem trimers, whose components are three hairpin subunits and two hinges. Our aim was to increase their selectivities to telomeres. After the optimization of SPPS, we synthesized the new tandem trimer Py-Im polyamide TT59, which targeted $18 \mathrm{bp}$ in the human telomere repetitive sequence TTAGGG. To our knowledge, $18 \mathrm{bp}$ is the longest specific binding sequence for Py-Im polyamides. Surface plasmon resonance (SPR) analysis was used to assess the binding affinity and specificity of new Py-Im polyamide TT59 toward telomeric repeat sequences. After conjugation of TH59 and TT59 with fluorescent 5-carboxytetramethylrhodamine (TAMRA), we obtained fluorescent telomere probes TAMRA TH59 and TAMRA TT59, respectively (Fig. 1). We found that the new tandem trimer probe TAMRA TT59 stained telomeres in chemically fixed cells and produced a lower background because of the reduction in nonspecific binding compared with the conventional probe TAMRA TH59. These results suggest that the new longer Py-Im polyamides recognize the target sequences in cells with greater specificity.

\section{Results and discussion}

\section{Synthesis of Py-Im Polyamides}

Based on the previous methodology using the synthesized building block 1 corresponding to $(R)$-2,4-diaminobutyric acid turn and three-ring cycle polyamide at the side of $\mathrm{N}$-terminal as one of the units, ${ }^{17 a}$ synthesis of fluorescent Py-Im polyamide probes was performed after optimization (Scheme 1). These

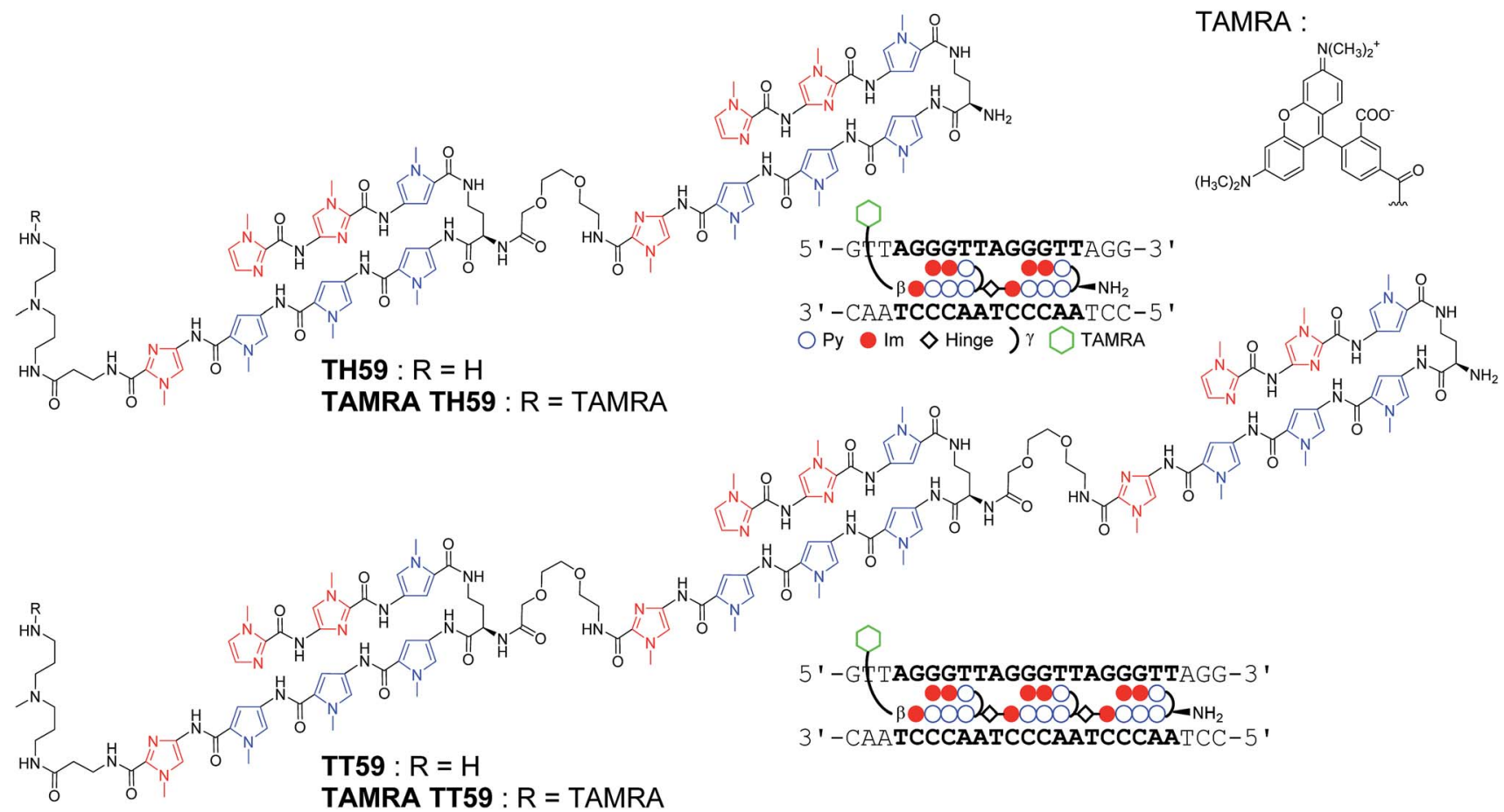

Fig. 1 Chemical structures of Py-Im polyamides TH59, TT59, TAMRA TH59 and TAMRA TT59 targeting the human telomere sequence and their ball-and-stick representations. 


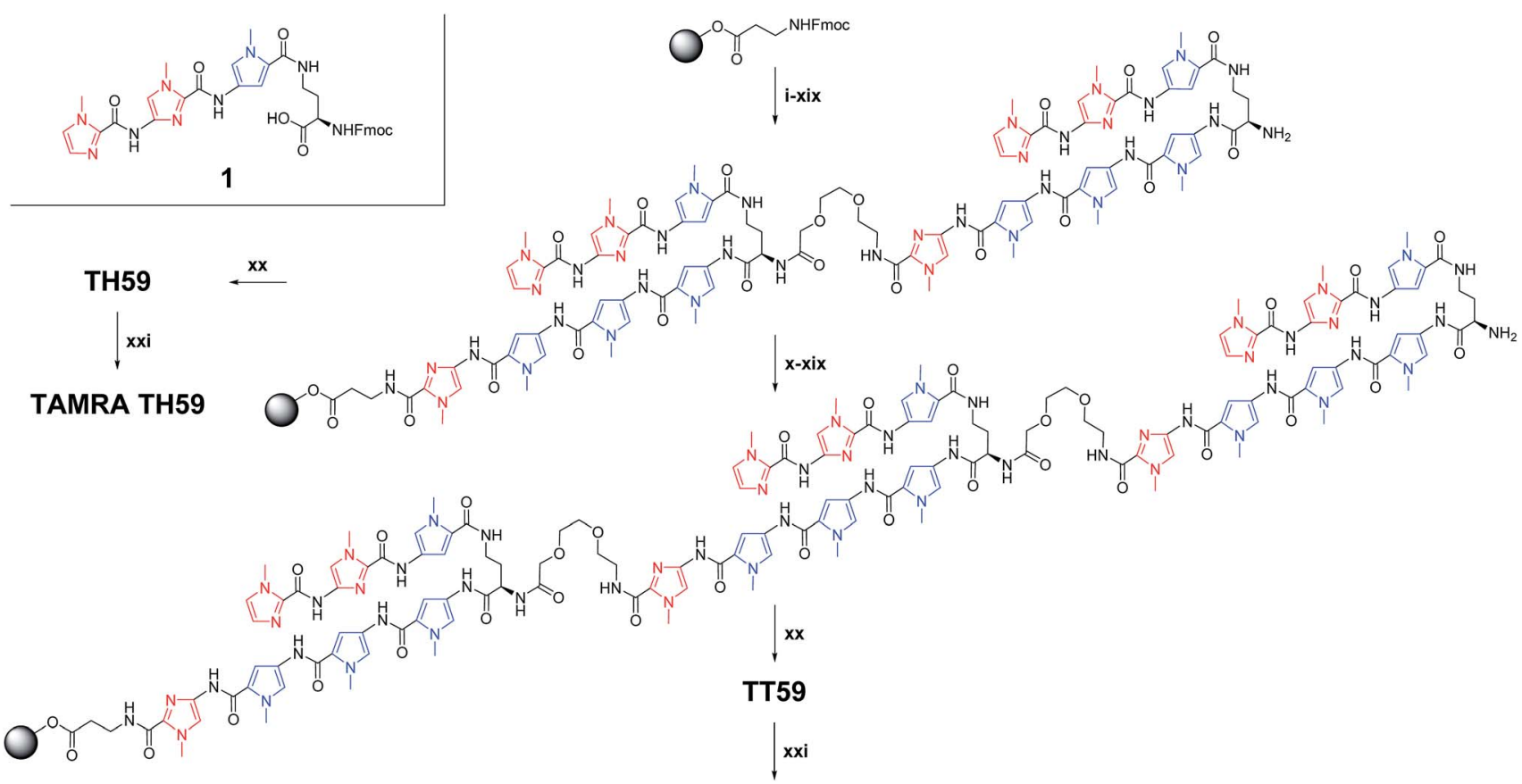

TAMRA TT59

Scheme 1 Solid-phase synthesis of Py-Im polyamides TH59, TT59, TAMRA TH59 and TAMRA TT59 and structure of building block 1. Reagents and conditions: (i) 20\% piperidine, NMP; (ii) Fmoc-Pylm- $\mathrm{CO}_{2} \mathrm{H}, \mathrm{HCTU}$, DIEA, NMP; (iii) 20\% piperidine, NMP; (iv) Fmoc-Py-CO $\mathrm{H}, \mathrm{HCTU}, \mathrm{DIEA}$, NMP; (v) 20\% piperidine, NMP; (vi) Fmoc-Py- $\mathrm{CO}_{2} \mathrm{H}$, HCTU, DIEA, NMP; (vii) 20\% piperidine, NMP; (viii) 1, HCTU, DIEA, NMP; (ix) 20\% piperidine, NMP; (x) Fmoc-mini-PEG-OH, HCTU, DIEA, NMP; (xi) 20\% piperidine, NMP; (xii) Fmoc-Pylm-CO ${ }_{2} \mathrm{H}, \mathrm{HCTU}$, DIEA, NMP; (xiii) 20\% piperidine, NMP; (xiv) Fmoc-Py- $\mathrm{CO}_{2} \mathrm{H}$, HCTU, DIEA, NMP; (xv) 20\% piperidine, NMP; (xvi) Fmoc-Py-CO ${ }_{2} \mathrm{H}, \mathrm{HCTU}$, DIEA, NMP; (xvii) 20\% piperidine, NMP; (xviii) 1, HCTU, DIEA, NMP; (xix) 20\% piperidine, NMP; (xx) 3,3'-diamino-N-methyldipropylamine, $55^{\circ} \mathrm{C}$; (xxi) 5-carboxytetramethylrhodamine, succinimidyl ester, DIEA, DMF.

syntheses have suffered from low yield, presumably due to the contamination of moisture. Therefore, the solid-phase peptide synthesizer was placed in a vinyl box with dehumidifiers. Machine-assisted Fmoc SPPS began from Fmoc- $\beta$-Ala-Wang resin. Each Fmoc group was deprotected 20\% piperidine/1methyl-2-pyrrolidone (NMP) and then the deprotected amino group reacted with the next Fmoc unit (Fmoc-Py-OH, FmocPyIm-OH, Fmoc-mini-PEG-OH or 1) activated with $N, N$-diisopropylethylamine (DIEA) and HCTU in NMP. Procedures (i)(xix) were performed for the synthesis of tandem dimer of hairpins on solid support. Though conventional TH59 could be obtained through the cleavage of the dimer from resin with $3,3^{\prime}$ diamino- $N$-methyldipropylamine, one repeat of procedures (x)(xix) was required for new tandem trimer TT59. As a result, the synthesis of tandem trimer Py-Im polyamide TT59 succeeded for the first time with an $8.5 \%$ yield for SPPS, and the yield of the previous tandem hairpin Py-Im polyamide TH59 increased from $6.9 \%$ (ref. $17 a$ ) to $29 \%$. The obtained Py-Im polyamides TH59 and TT59 were conjugated with 5-carboxytetramethylrhodamine succinimidyl ester in $N, N$-dimethylformamide (DMF) and DIEA to afford the fluorescent Py-Im polyamide probes TAMRA TH59 and TAMRA TT59, respectively. Protection of the $\alpha$-amino group of Dab was not required because of steric hindrance. The ESI-TOF MS spectra and analytical HPLC profiles of TH59, TT59, TAMRA TH59 and TAMRA TT59 are shown in Fig. S1 and S2, $\dagger$ respectively.

\section{Optical characteristics of the fluorescent probes}

The UV-visible absorption spectra of probes TAMRA TH59 and TAMRA TT59 are shown in Fig. S3A. $\dagger$ The absorption maximum at $\sim 555 \mathrm{~nm}$ was due to the fluorescent group TAMRA. The absorption maximum at $\sim 310 \mathrm{~nm}$ was derived from the $\mathrm{N}$-methylpyrrole and $\mathrm{N}$-methylimidazole moieties.

Fig. S3B $\uparrow$ shows the fluorescence spectra of probes TAMRA TH59 and TAMRA TT59 in the absence and presence of 1.0 or 2.0 equivalent oligonucleotide (ODN)-1/2, whose sequences are shown in Table S1. $\dagger$ Addition of ODN-1/2 to both probes provided little change in the fluorescence intensity and quench of TAMRA was not observed in the absence of ODN-1/2. In contrast, Dervan, Gray and co-workers showed that TAMRA connected to $N$-methylpyrrole in the polyamide backbone was quenched because of the electron transfer from Py to TAMRA in the absence of dsDNA. ${ }^{18}$ It is presumed that the distance between TAMRA attaching to C-terminal through 3,3'-diamino$N$-methyldipropylamine linker and polyamide was longer and therefore the electron transfer triggering quench became difficult to occur.

\section{Binding affinities to match sequence and discrimination of mismatch sequences}

Binding affinity and discrimination of TH59 and TT59 were evaluated with SPR experiments. ${ }^{17 b, 19}$ Three kinds of 
5'-biotinylated hairpin DNAs ODN-3, 4 and 5 containing the binding site of TT59 were prepared by the immobilization to sensor chips through a biotin-avidin system. The sequences are shown in Table 1 and S1. $\dagger$ ODN-3, 4 and 5 had no mismatch, 1 bp mismatch at the C-terminal of TT59 and 1 bp mismatch at the center of TT59, respectively. Tandem hairpin TH59 and trimer TT59 were passed to DNAs on sensor chips and resulting sensorgrams (shown in Fig. S4 and $5 \dagger$ ) and values (summarized in Table 1) were obtained. Comparing the values of TT59 in the matching sequence with those of conventional tandem hairpin TH59, the association of TT59 was slower and thus its binding affinity was weaker. One considerable reason was that bigger structure of tandem trimer TT59 decreased its own accessibility to the matching sequence. However, since the $K_{\mathrm{D}}$ value of TT59 and ODN-3 was $3.6 \times 10^{-9} \mathrm{M}$, the tandem trimer had high binding affinity comparable with other Py-Im polyamides. ${ }^{7}$ To evaluate the discriminations, specificities were calculated by dividing the $K_{\mathrm{D}}$ value of the mismatch DNA (ODN-4 or 5) by that of the match DNA (ODN-3). The specificity value of tandem trimer TT59 was bigger than that of TH59, suggesting that extending the targeted base pairs could increase the sequence specificities.

\section{Human telomere staining with two Py-Im polyamide probes}

We doubly stained human HeLa 1.3 cell spreads, which are used for clinical karyotype tests, with 4',6-diamidino-2-phenylindole (DAPI) and Py-Im polyamide probes TAMRA TH59 and TAMRA TT59 to compare their abilities to stain telomeres selectively. Cell spreads were prepared by methanol/acetic acid fixation. To ensure a proper comparison between TAMRA TH59 and TAMRA TT59, the images without deconvolution, differently from our previous study, ${ }^{17 a}$ were presented in Fig. 2. Furthermore, to demonstrate reproducibility of the result, additional image data was depicted in Fig. S6. $\dagger$ DAPI visualized chromosomal regions and nuclei, and Py-Im polyamide probes showed intense foci.
(A) TAMRA TH59

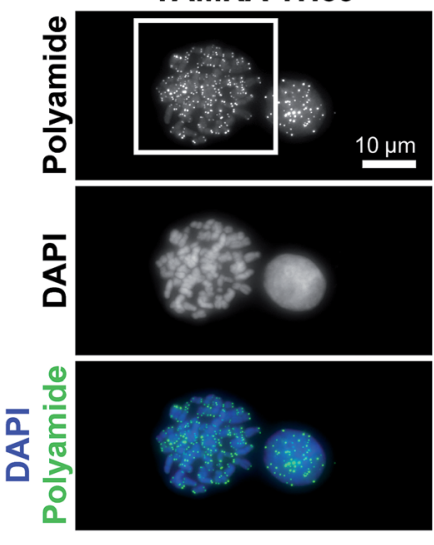

(B)

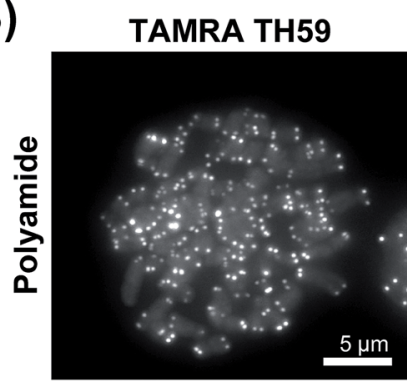

Fig. 2 Telomere staining of HeLa 1.3 cell spreads with fluorescent polyamides. (A) The HeLa 1.3 cell spread was stained with the fluorescent polyamide (first row) and DAPI (second row). The merged images are shown in the third row. The first and second columns show the results for TAMRA TH59 and TAMRA TT59, respectively. Enlarged images of the boxed regions in panel (A) are shown in panel (B).

Two foci were observed at every chromosomal end, indicating that both probes could stain telomeres selectively. However, the signal intensities appeared to be greater for the probe TAMRA

Table 1 Binding affinities of polyamides TH59 and TT59 against match sequence (ODN-3) or 1 bp mismatch sequence (ODN-4 or 5) ${ }^{a}$

\begin{tabular}{|c|c|c|c|c|c|c|c|c|c|c|}
\hline & \multicolumn{5}{|c|}{ TH59 } & \multicolumn{5}{|c|}{ TT59 } \\
\hline & $\begin{array}{c}k_{\mathrm{a}} \\
\left(\mathrm{M}^{-1} \mathrm{~s}^{-1}\right)\end{array}$ & $k_{\mathrm{d}}\left(\mathrm{s}^{-1}\right)$ & $K_{\mathrm{D}}(\mathrm{M})$ & $\chi^{2}$ & Specificity & $\begin{array}{c}k_{\mathrm{a}} \\
\left(\mathrm{M}^{-1} \mathrm{~s}^{-1}\right)\end{array}$ & $k_{\mathrm{d}}\left(\mathrm{s}^{-1}\right)$ & $K_{\mathrm{D}}(\mathrm{M})$ & $\chi^{2}$ & Specificity \\
\hline $\begin{array}{c}\text { 5'-biotin-GGTTAGAGTTAGGGTTAGGGTTAGG } \\
\text { 3'-CCAATCTCAATCCCAATCCCAATCC }_{T_{T}} \\
\text { ODN-4 }\end{array}$ & $3.4 \times 10^{5}$ & $6.5 \times 10^{-4}$ & $1.9 \times 10^{-9}$ & 0.36 & 1.1 & $5.3 \times 10^{4}$ & $4.9 \times 10^{-4}$ & $9.3 \times 10^{-9}$ & 0.60 & 2.6 \\
\hline $\begin{array}{c}\text { 5'-biotin-GGTTAGGGTTAGAGTTAGGGTTAGG } \\
\text { 3'-CCAATCCCAATCTCAATCCCAATCC }{ }_{T_{T}}^{\frac{T}{T}} \\
\text { ODN-5 }\end{array}$ & $1.3 \times 10^{5}$ & $3.7 \times 10^{-4}$ & $2.8 \times 10^{-9}$ & 0.20 & 1.6 & $4.7 \times 10^{4}$ & $4.2 \times 10^{-4}$ & $9.0 \times 10^{-9}$ & 0.33 & 2.5 \\
\hline
\end{tabular}


TH59 (ref. 17b) than for TAMRA TT59, presumably because more TAMRA TH59 molecules bound to telomeres compared with TAMRA TT59. On the other hand, as shown in Fig. 2B, substantial polyamide signals were observed on the chromosome body when treating cells with TAMRA TH59. By contrast, lower-intensity signals on the chromosome body region were observed in the case of TAMRA TT59. Background polyamide signals except intense foci derived from nonspecific binding. Cell spreads images indicated TAMRA TT59 had higher selectivity to telomeres, suggesting that nonspecific binding was reduced by increasing the number of base pairs bound by Py-Im polyamides.

This result was supported by telomere staining images of HeLa 1.3 cells fixed in formaldehyde (Fig. 3 and S7†), a commonly used fixation method in cell biology. Many sharp polyamide signals were detected by staining with both TAMRA TH59 and TAMRA TT59 in nuclei. By contrast, throughout the nucleus, TAMRA TT59 signals were less intense and rarely merged with DAPI signals. For quantification of the telomere signals, they were extracted from Fig. 3 non-deconvolved images and the results are shown in Fig. 4 as surface plots and signal-

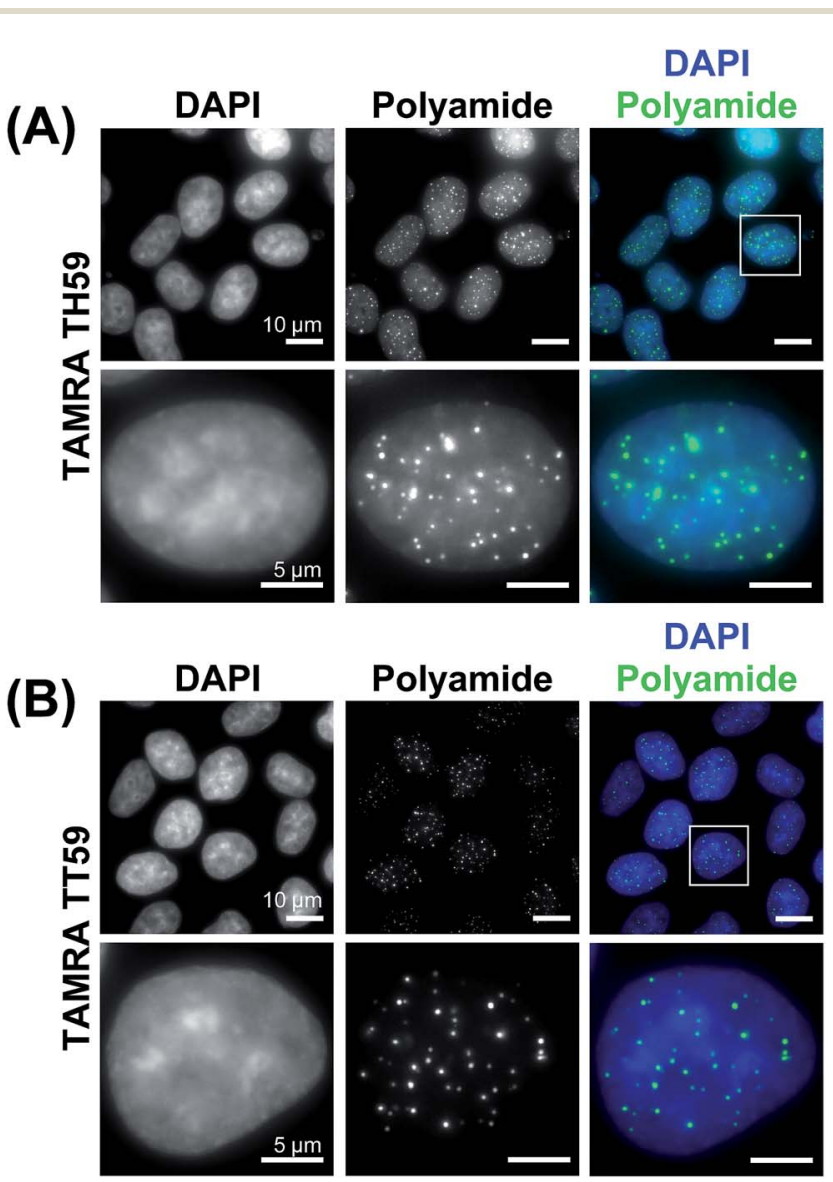

Fig. 3 Telomere staining of HeLa 1.3 cells with fluorescent polyamides. HeLa 1.3 cells were stained with DAPI (first column) and fluorescent polyamides (second column). The merged images are shown in the third column. Enlarged images of the boxed regions in the first row are shown in the second row. Cell images using TAMRA TH59 and TAMRA TT59 are shown in panels (A) and (B), respectively.
(A)

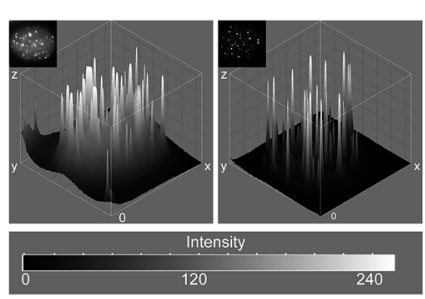

(B)

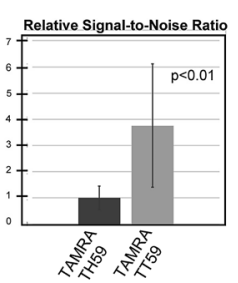

Fig. 4 (A) Surface plots based on the boxed images shown in Fig. 3. (B) Relative signal-to-noise ratios of the images.

to-noise ratio. The sharper surface plots and about 3.7-fold signal-to-noise ratio compared to TAMRA TH59 also showed lower-intensity background signals of TAMRA TT59, suggesting its higher selectivity to telomeres. These images of cell staining were supported by the results of SPR analyses.

\section{Conclusion}

In this study, we have shown the new motif of Py-Im polyamide, which we call a tandem trimer, which comprises three hairpins and two hinge segments and targets $18 \mathrm{bp}$, the longest reported sequence for Py-Im polyamides. SPR analysis indicated that the tandem trimer Py-Im polyamide bound to $18 \mathrm{bp}$ in human telomere repeats more specifically than did the previous tandem hairpin Py-Im polyamides. Moreover, the TAMRAconjugated tandem trimer Py-Im polyamide probe highlighted the telomere foci clearly with lower intensity of background signals, showing its higher selectivity to telomeres. This new motif may be useful for highlighting specific regions clearly in human cells.

\section{Acknowledgements}

We thank Dr T. de Lange (Rockefeller University) for the gift of HeLa 1.3 cells and Mr S. Asamitsu (Kyoto University) for assistance in SPR data analysis. This work was supported by NIG collaboration grant, MEXT grant, and JST CREST.

\section{Notes and references}

1 E. H. Blackburn, Angew. Chem., Int. Ed., 2010, 49, 7405-7421.

2 (a) J. Nandakumar and T. R. Cech, Nat. Rev. Mol. Cell Biol., 2013, 14, 69-82; (b) G. Biffi, D. Tannahill, J. McCafferty and S. Balasubramanian, Nat. Chem., 2013, 5, 182-186; (c) Y. Doksani, J. Y. Wu, T. de Lange and X. Zhuang, Cell, 2013, 155, 345-356.

3 V. A. Zakian, Exp. Cell Res., 2012, 318, 1456-1460.

4 A. Smogorzewska and T. de Lange, Annu. Rev. Biochem., 2004, 73, 177-208.

5 A. Smogorzewska, B. van Steensel, A. Bianchi, S. Oelmann, M. R. Schaefer, G. Schnapp and T. de Lange, Mol. Cell. Biol., 2000, 20, 1659-1668.

6 (a) A. S. Boutorine, D. S. Novopashina, O. A. Krasheninina, K. Nozeret and A. G. Venyaminova, Molecules, 2013, 18, 15357-15397; (b) P. M. Lansdorp, N. P. Verwoerd, F. M. van 
de Rijke, V. Dragowska, M. T. Little, R. W. Dirks, A. K. Raap and H. J. Tanke, Hum. Mol. Genet., 1996, 5, 685-691.

7 (a) J. W. Trauger, E. E. Baird and P. B. Dervan, Nature, 1996, 382, 559-561; (b) S. White, J. W. Szewczyk, J. M. Turner, E. E. Baird and P. B. Dervan, Nature, 1998, 391, 468-471; (c) P. B. Dervan, Bioorg. Med. Chem., 2001, 9, 2215-2235; (d) P. B. Dervan and B. S. Edelson, Curr. Opin. Struct. Biol., 2003, 13, 284-299; (e) P. B. Dervan, R. M. Doss and M. A. Marques, Curr. Med. Chem.: Anti-Cancer Agents, 2005, 5, 373-387; $(f)$ M. S. Blackledge and C. Melander, Bioorg. Med. Chem., 2013, 21, 6101-6114.

8 (a) D. M. Herman, E. E. Baird and P. B. Dervan, J. Am. Chem. Soc., 1998, 120, 1382-1391; (b) S. E. Swalley, E. E. Baird and P. B. Dervan, J. Am. Chem. Soc., 1999, 121, 1113-1120.

9 N. R. Wurtz, J. M. Turner, E. E. Baird and P. B. Dervan, Org. Lett., 2001, 3, 1201-1203.

10 (a) J. W. Trauger, E. E. Baird, M. Mrksich and P. B. Dervan, J. Am. Chem. Soc., 1996, 118, 6160-6166; (b) J. W. Trauger, E. E. Baird and P. B. Dervan, J. Am. Chem. Soc., 1998, 120, 3534-3535.

11 (a) M. Mrksich, M. E. Parks and P. B. Dervan, J. Am. Chem. Soc., 1994, 116, 7983-7988; (b) R. P. L. de Clairac, B. H. Geierstanger, M. Mrksich, P. B. Dervan and D. E. Wemmer, J. Am. Chem. Soc., 1997, 119, 7909-7916.

12 (a) D. M. Herman, J. M. Turner, E. E. Baird and P. B. Dervan, J. Am. Chem. Soc., 1999, 121, 1121-1129; (b) D. M. Chenoweth and P. B. Dervan, J. Am. Chem. Soc., 2010, 132, 14521-14529; (c) H. Morinaga, T. Bando, T. Takagaki, H. Yamamoto, K. Hashiya and H. Sugiyama, J. Am. Chem. Soc., 2011, 133, 18924-18930; (d) B. C. Li, D. C. Montgomery, J. W. Puckett and P. B. Dervan, J. Org. Chem., 2013, 78, 124-133.

13 (a) T. Bando and H. Sugiyama, Acc. Chem. Res., 2006, 39, 935944; (b) X. Xiao, P. Yu, H. S. Lim, D. Sikder and T. Kodadek, Angew. Chem., Int. Ed., 2007, 46, 2865-2868; (c) J. L. Meier, A. S. Yu, I. Korf, D. J. Segal and P. B. Dervan, J. Am. Chem. Soc., 2012, 134, 17814-17822; (d) T. Vaijayanthi, T. Bando,
G. N. Pandian and H. Sugiyama, ChemBioChem, 2012, 13, 2170-2185; (e) I. Singh, C. Wendeln, A. W. Clark, J. M. Cooper, B. J. Ravoo and G. A. Burley, J. Am. Chem. Soc., 2013, 135, 3449-3457; (f) G. N. Pandian, J. Taniguchi, S. Junetha, S. Sato, L. Han, A. Saha, C. Anandhkumar, T. Bando, H. Nagase, T. Vaijayanthi, R. D. Taylor and H. Sugiyama, Sci. Rep., 2014, 4, 3843.

14 (a) P. Weyermann and P. B. Dervan, J. Am. Chem. Soc., 2002, 124, 6872-6876; (b) A. T. Poulin-Kerstien and P. B. Dervan, J. Am. Chem. Soc., 2003, 125, 15811-15821; (c) M. Yamamoto, T. Bando, H. Morinaga, Y. Kawamoto, K. Hashiya and H. Sugiyama, Chem.-Eur. J., 2014, 20, 752-759.

15 (a) D. M. Herman, E. E. Baird and P. B. Dervan, Chem.-Eur. J., 1999, 5, 975-983; (b) I. Kers and P. B. Dervan, Bioorg. Med. Chem., 2002, 10, 3339-3349; (c) T. D. Schaal, W. G. Mallet, D. L. McMinn, N. V. Nguyen, M. M. Sopko, S. John and B. S. Parekh, Nucleic Acids Res., 2003, 31, 1282-1291; (d) S. Sasaki, T. Bando, M. Minoshima, K. Shinohara and H. Sugiyama, Chem.-Eur. J., 2008, 14, 864-870.

16 K. Maeshima, S. Janssen and U. K. Laemmli, EMBO J., 2001, 20, 3218-3228.

17 (a) Y. Kawamoto, T. Bando, F. Kamada, Y. Li, K. Hashiya, K. Maeshima and H. Sugiyama, J. Am. Chem. Soc., 2013, 135, 16468-16477; (b) A. Hirata, K. Nokihara, Y. Kawamoto, T. Bando, A. Sasaki, S. Ide, K. Maeshima, T. Kasama and H. Sugiyama, J. Am. Chem. Soc., 2014, 136, 11546-11554; (c) M. Yamamoto, T. Bando, Y. Kawamoto, R. D. Taylor, K. Hashiya and H. Sugiyama, Bioconjugate Chem., 2014, 25, 552-559; (d) R. D. Taylor, Y. Kawamoto, K. Hashiya, T. Bando and H. Sugiyama, Chem.-Asian J., 2014, 9, 25272533.

18 V. C. Rucker, A. R. Dunn, S. Sharma, P. B. Dervan and H. B. Gray, J. Phys. Chem. B, 2004, 108, 7490-7494.

19 E. R. Lacy, N. M. Le, C. A. Price, M. Lee and W. D. Wilson, J. Am. Chem. Soc., 2002, 124, 2153-2163. 